\title{
III-V/Silicon First Order Distributed Feedback Lasers Integrated on SOI Waveguide Circuits
}

\author{
S. Keyvaninia ${ }^{(1)}$, S. Verstuyft ${ }^{(1)}$, L. Van Landschoot ${ }^{(1)}, D$. Van Thourhout ${ }^{(1)}$, G. Roelkens ${ }^{(1)}, G-. H$. \\ Duan, F. Lelarge ${ }^{(2)}$, J.-M. Fedeli ${ }^{(3)}$, S. Messaoudene ${ }^{(3)}{ }^{(4)}$ T. De Vries ${ }^{(4)}$, E.J. Geluk ${ }^{(4)}$, B. Smalbrugge ${ }^{(4)}$, \\ M. Smit ${ }^{(4)}$
}

(1) Photonics Research Group, INTEC, Ghent University-IMEC, B-9000 Gent, Belgium,

(2) III-V Lab, a joint lab of 'Alcatel-Lucent Bell Labs France', 'Thales Research and Technology' and 'CEA Leti', Campus Polytechnique, 1, Avenue A. Fresnel, 91767 Palaiseau cedex, France.

(3) CEA-LETI, Minatec Campus, 17 Rue des Martyrs, 38054 Grenoble, France.

(4) COBRA Research Institute, Eindhoven University of Technology (TU/e) Eindhoven, The

Netherlands.

\section{shahram.keyvaninia@intec.ugent.be}

Abstract Heterogeneously integrated III-V-on-silicon first order distributed feedback lasers utilizing an ultra-thin DVS-BCB die-to-wafer bonding process are reported. A novel design exploiting high confinement in the active waveguide is demonstrated. $5 \mathrm{~mW}$ output power coupled to a silicon waveguide, $40 \mathrm{~dB}$ side mode suppression ratio and continuous wave operation up to $60^{\circ} \mathrm{C}$ is obtained.

\section{Introduction}

Silicon photonics is clearly becoming an enabling technology for the realization of integrated optical transceivers for optical interconnect applications. The key component that is difficult to realize in this technology however is the laser source. Such a source needs to provide single wavelength emission with milliwatt level optical output power and should be able to operate at elevated temperatures. A monolithically integrated laser would be the ultimate solution, and a substantial body of research is geared towards the implementation of germanium lasers [1]. However, the performance of these sources does not yet match the typical performance of a III-V semiconductor laser diode. Therefore, several research groups focus on the heterogeneous integration of III-V semiconductor layer stacks on silicon waveguide circuits. This can be realized through either molecular wafer bonding or adhesive wafer bonding [2]. Several single-wavelength laser types have been demonstrated in literature, including distributed Bragg reflector lasers $[2,3]$ and distributed feedback lasers $[2,4]$. These laser structures typically use a hybrid laser waveguide geometry, where the bulk of the optical power is concentrated in the silicon device layer, while the tail of the evanescent mode feels the gain from the multi-quantum well gain region. In this paper we report on distributed feedback lasers integrated on a silicon waveguide circuit, where the bulk of the optical mode is concentrated in the III-V waveguide layer and the mode tail overlaps with the silicon device layer, in which the first order quarter wave shifted Bragg grating is

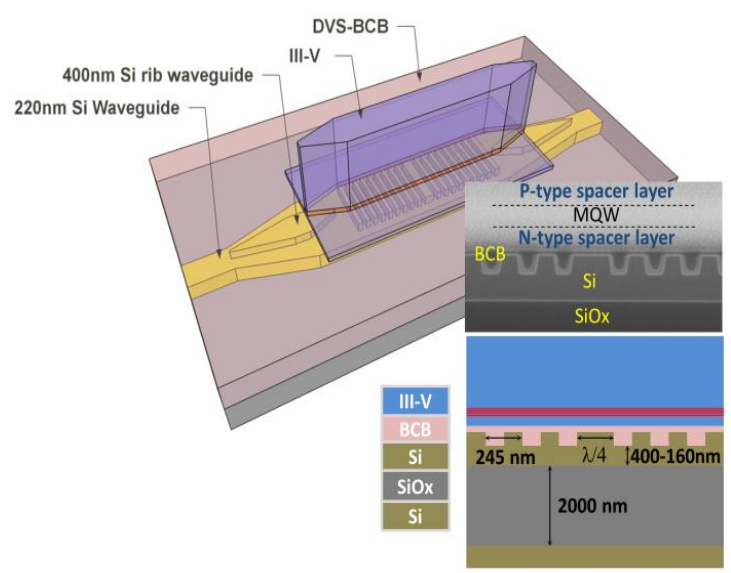

Fig. 1: Schematic of the heterogeneously integrated first order distributed feedback laser presented in this work

implemented. This gives rise to higher modal gain, but necessitates the use of a spotsize converter to efficiently couple the laser emission to the underlying silicon waveguide circuit.

\section{Device Design}

The device design is schematically depicted in Figure 1, showing the III-V thin film laser mesa bonded onto a silicon waveguide circuit using an adhesive bonding layer (DVS-BCB). The silicon waveguide circuit consists of a $400 \mathrm{~nm}$ thick crystalline silicon device layer that is used to define the quarter wave shifted first order distributed feedback grating structures $(180 \mathrm{~nm}$ deep etch) and to allow realizing an efficient spotsize converter between the III-V device layer and the silicon device layer. After the laser emission is coupled to the $400 \mathrm{~nm}$ silicon device layer a second spotsize converter structure is 
used to couple to a $220 \mathrm{~nm}$ thick device layer thickness, since this device layer thickness is emerging as a standard in different silicon photonics foundry services and allows the implementation of high performance passive and electro-optic functionality. In order to obtain a high efficiency power transfer between the III$\checkmark$ device layer and the silicon waveguide layer, a piecewise linear taper structure was implemented in both layers, where the III-V taper first quickly $(L=35 \mu \mathrm{m})$ tapers from the laser mesa width of $3 \mu \mathrm{m}$ to $0.9 \mu \mathrm{m}$, after which a second linear taper of $180 \mu \mathrm{m}$ long tapers down to a III-V taper tip width of $500 \mathrm{~nm}$. At the same time the silicon waveguide structure underneath tapers from $300 \mathrm{~nm}$ to $1000 \mathrm{~nm}$ over $180 \mu \mathrm{m}$ length. The III-V layer stack that is used for the device demonstration consists of a 200 $\mathrm{nm}$ thick n-InP contact layer, two $100 \mathrm{~nm}$ thick InGaAsP separate confinement heterostructure layers (bandgap wavelength $1.17 \mu \mathrm{m}$ ), 6 InGaAsP quantum wells (6 $\mathrm{nm}$ thick, emission wavelength $1.55 \mu \mathrm{m}$ ) surrounded by InGaAsP barriers, a $1.5 \mu \mathrm{m}$ thick $\mathrm{p}$-InP top cladding and a $100 \mathrm{~nm} \mathrm{p++}$ InGaAs contact layer. The confinement factor of the optical mode in the 6 quantum wells is $9.3 \%$. Since the taper is implemented in the same epitaxial layer stack as the laser mesa it also needs to be electrically pumped in order to prevent excessive absorption in the spot size converter.

\section{Device Fabrication}

The silicon device wafer fabrication was carried out in a CMOS pilot line on $200 \mathrm{~mm}$ SOI wafers. Three etch steps were used (180 nm deep, 70 $\mathrm{nm}$ deep and $220 \mathrm{~nm}$ deep) for the definition of the waveguide structures. The $70 \mathrm{~nm}$ etch depth is used to define the fiber-to-chip grating coupler structures. After waveguide etching, a SiO2 top cladding was applied, after which chemical mechanical polishing was used to planarize the wafer. The III-V layer stack was bonded onto the silicon device layer using a $100 \mathrm{~nm}$ thick DVS$\mathrm{BCB}$ adhesive bonding layer, after which the InP substrate was removed [4]. The laser mesa was defined using i-line contact lithography and wet etching. The required $500 \mathrm{~nm}$ III-V taper tip widths were successfully obtained this way. After etching through the multi-quantum well layers, the n-type contacts were defined, the mesa is passivated and $p$-contact metallization is applied. A scanning electron microscopy picture of the fabricated taper tips is shown in Figure 2.

\section{Device Characterization}

First order quarter wave shifted devices with a

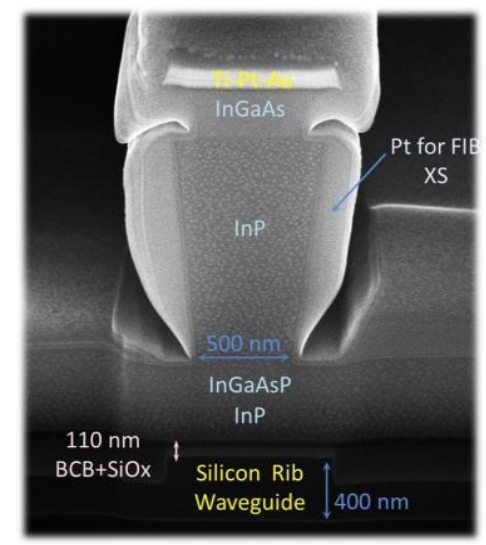

Fig. 2: Scanning electron microscope picture of the III-V semiconductor taper tip realized using i-line lithography and wet etching.

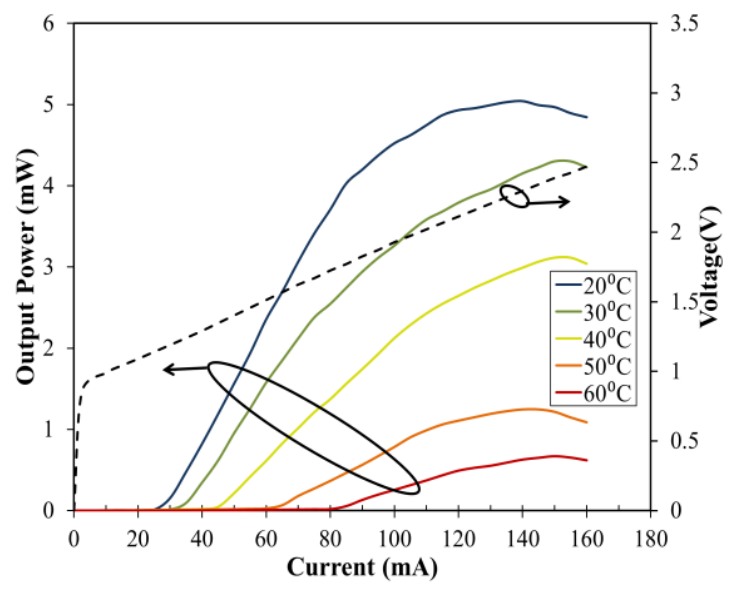

Fig. 3: Laser L-I-V characteristics.

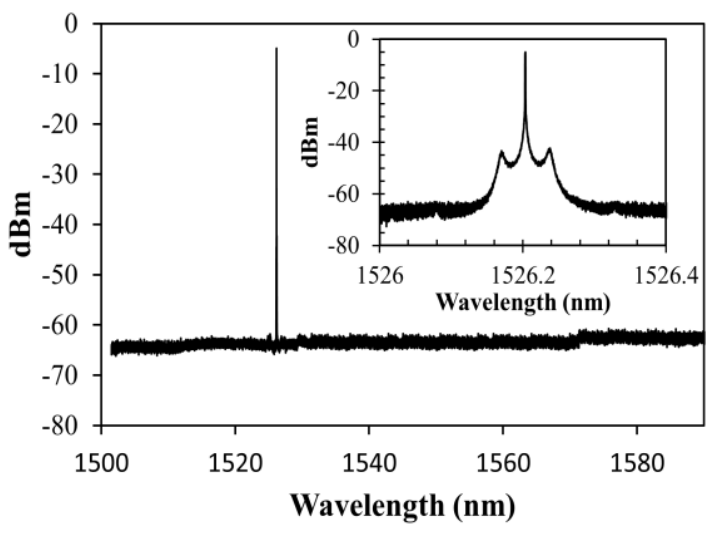

Fig. 4: The laser emission spectrum.

grating period of $245 \mathrm{~nm}$ and a total grating length of $600 \mu \mathrm{m}$ were characterized. For a 100 $\mathrm{nm}$ DVS-BCB bonding layer thickness the grating coupling strength is simulated to be 20 $\mathrm{cm}^{-1}$. The laser device characterization was carried out from room temperature up to $60^{\circ} \mathrm{C}$. The resulting L-I-V curves are shown in Figure 
3 , illustrating continuous wave operation up to $60^{\circ} \mathrm{C}$ and over $5 \mathrm{~mW}$ optical output power coupled to the silicon device layer. The laser threshold is $29 \mathrm{~mA}$ at room temperature and the external differential efficiency is $0.072 \mathrm{~A} / \mathrm{W}$. The series resistance of the laser is $9 \Omega$. The laser spectrum is shown in Figure 4. More than $40 \mathrm{~dB}$ side mode suppression ratio was obtained. The laser linewidth was characterized using a delayed self-heterodyne measurement setup using $22.5 \mathrm{~km}$ of optical fiber. Typical laser linewidths of $1 \mathrm{MHz}$ are obtained. This shows that these devices have satisfying characteristics to be used in silicon-based transceiver technology.

\section{Conclusions}

In this paper we presented first order distributed feedback lasers heterogeneously integrated on a silicon device wafer. While currently the silicon device layer only contains passive optical functionality, one can envisage connecting arrays of such distributed feedback lasers to a bench of silicon optical modulators and wavelength multiplexers to realize a high aggregate bitrate silicon photonics transmitter module.

\section{Acknowledgements}

This work was carried out in the framework of the FP7-ICT-HELIOS integrated project. The authors would like to thank Lemos Alvares Dos Santos and Saeed Tahvili for the assistance in the laser linewidth measurements.

\section{References}

[1] R. E. Camacho-Aguilera, et al., Optics Express, 20, 10 (2012).

[2] G. Roelkens, et al., Laser Photonics Rev. P.DOI: 10.1002/lpor.200900033 (2010).

[3] S. Keyvaninia, et al., Optics Express, 21, 3 (2013).

[4] S. Stankovic, et al., IEEE Photonics Technology Letters, 24, 23, (2012)

[5] S. Keyvaninia, et al., Optical Materials Express, 3, 1 (2013) 
